

Entrevista

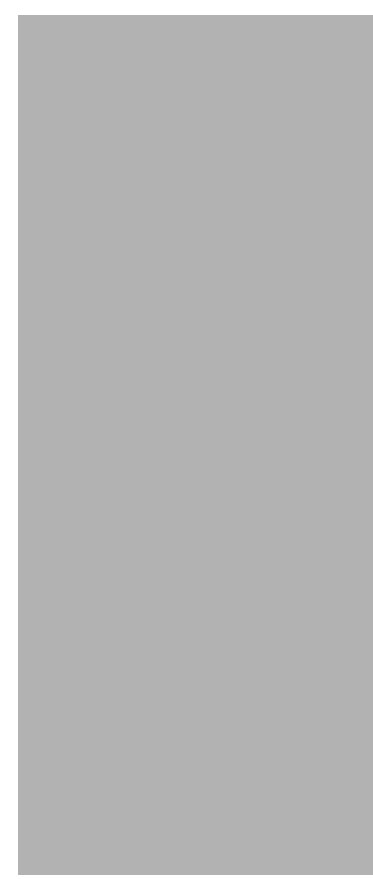





\title{
¿QUÉ SIGNIFICA DESCOLONIZAR EUROPA? HISTORIA, CULTURA Y \\ DESCOLONIZACIÓN DE LAS CIENCIAS \\ SOCIALES. ENTREVISTA A MARÍA PAULA MENESES ${ }^{\prime}$
}

\section{WHAT WOULD IT MEAN TO DECOLONIZE EUROPE? HISTORY, CULTURE AND SOCIAL SCIENCES DECOLONIZATION. INTERVIEW TO MARÍA PAULA MENESES}

\author{
Javier García Fernández \\ Universidad de Coimbra, Coimbra, Portugal \\ j.garcia.fer1987@gmail.com
}

Palabras clave: Historia, colonialismo, ciencias sociales, eurocentrismo.

Keywords: History, colonialism, social sciences, eurocentrism.

Resumen: La entrevista abre un diálogo desde Andalucía (el sur del Estado español) con la profesora María Paula Meneses en torno a los legados de los proyectos imperiales, los procesos de descolonización y los distintos saberes nacidos de las luchas anti-coloniales. Abordamos las siguientes cuestiones: cómo podríamos pensar históricamente desde el sur y qué significa descolonizar Europa. Además, exploramos los lugares desde los que se podrían construir ciencias sociales no eurocéntricas.

Abstract: The following interview aims to open a dialogue from Andalusia (In the South of the Iberian Peninsula) with Professor María Paula Meneses about the legacies of several imperial projects, decolonization processes and different epistemologies born after anti-colonial struggles. The interview is structured around a central question: how could we think historically from the South and what it means to decolonize Europe? In addition, we explore the places from which non-Eurocentric social sciences could be thought.

1. Entrevista realizada por Javier García Fernández a la profesora María Paula Meneses en los jardines de la Facultad de Filosofía y Letras de la Universidad de Granada en el contexto del IV Congreso de Antropólogos Iberoamericanos en Red, los días 5, 6 y 7 de septiembre de 2018. La corrección técnica y la revisión ha sido llevaba a cabo por Ígor Rodríguez-Iglesias a quien agradezco de corazón sus comentarios en el proceso de transcripción de la entrevista oral a la escritura. 


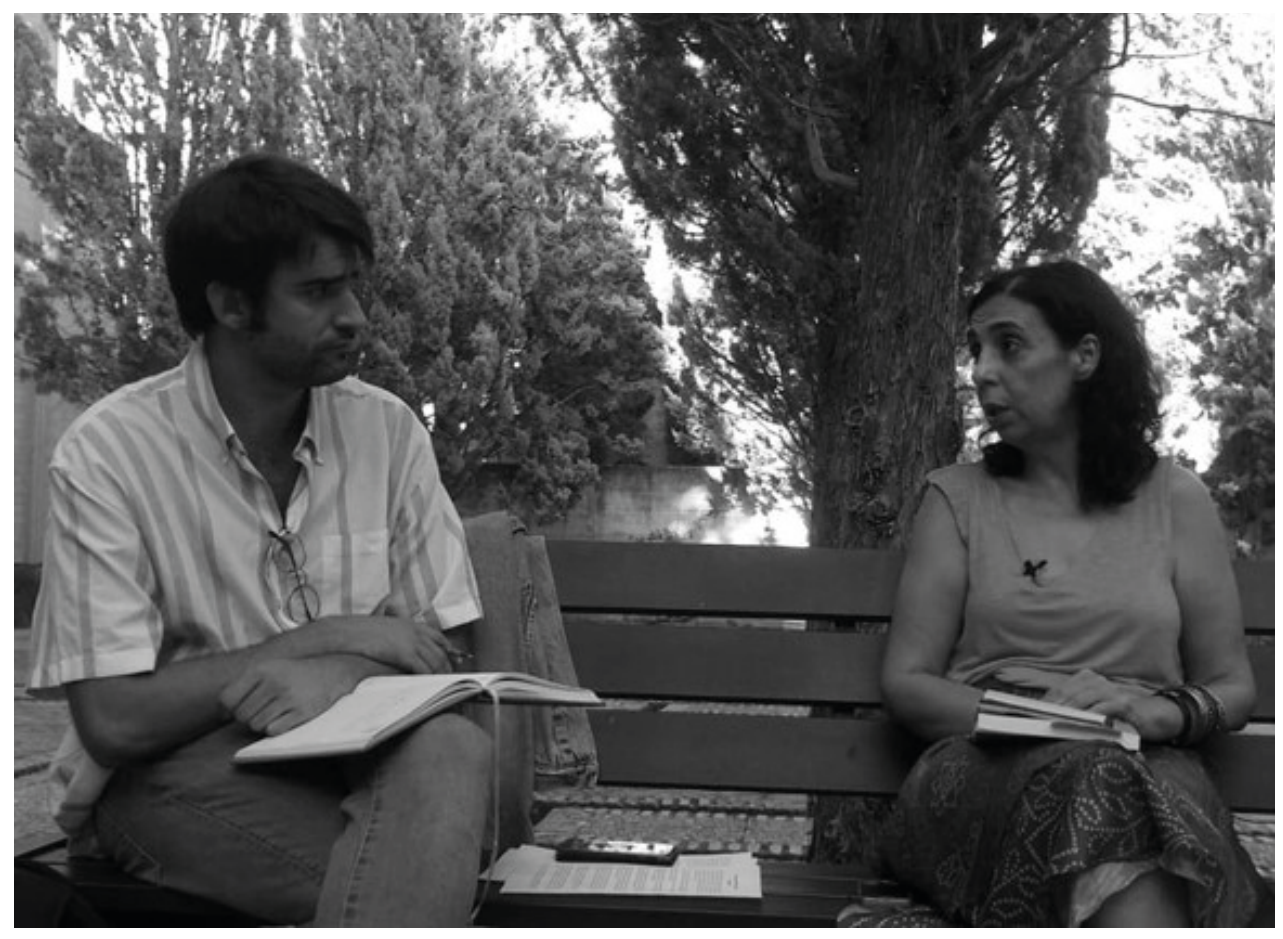

La profesora María Paula Meneses es mozambiqueña, antropóloga e historiadora de la Universidad de Coimbra. Formada académicamente en la Unión Soviética, obtuvo su doctorado en la Universidad de Rutgers. Ha sido docente universitaria en la Universidad Eduardo Mondlane y, a partir de los años noventa, en la Universidad de Coimbra, donde es profesora actualmente y vicepresidenta científica del Centro de Estudios Sociales. Es coordinadora junto a los profesores Boaventura de Sousa Santos y Antonio Sousa Ribeiro, del Programa de Doctorado en Poscolonialismos y Ciudadanía Global. También coordina junto a Boaventura de Sousa Santos y a Karina Bidaseca el Curso Internacional de especialización en Epistemologías del Sur, desarrollado por el Consejo Latinoamericano de Ciencias Sociales (CLACSO). Ha desarrollado, junto a Boaventura Sousa Santos y el equipo de investigadores del CES, durante la última década la propuesta intelectual de las epistemologías del sur. Hoy participa en distintos foros, como teórica y pensadora y como articulista en la prensa, en los debates sobre democracia en África, memorias africanas y otros modelos de desarrollo y justicia para África. Sus últimos libros publicados han sido Epistemologías del Sur: Perspectivas, coeditado junto a Boaventura de Sousa Santos, y Mozambique on the Move: Challenges and Reflections, coeditado junto a Sheila Khan and Bjorn Bertelsen. Se trata de una de las intelectuales de referencia en el contexto internacional en el área de los procesos de descolonización y la relación entre poder, conocimiento y sociedades africanas. Es también una de las mayores especialistas internacionales en pluralismo jurídico, culturas africanas y salud comunitaria en el contexto de África del sur. 
PREGUNTA: ¿Cuéntanos algo sobre Mozambique, la lucha de liberación y el contexto político en que te formaste? ¿Cómo viviste la descolonización?

Buenas tardes, muchas gracias por la invitación para dialogar sobre nuestras realidades. Bueno, para entender lo que significó para mí la descolonización de Mozambique, y para mostrar que la Historia siempre tiene varias interpretaciones, tenemos que partir de las guerras de liberación en Guinea Bisau, Angola y Mozambique, que llevan al 25 de Abril en Portugal, lo cual desemboca en un golpe de Estado que conduce directamente a un proceso de cambio político en Portugal. La primera cuestión sería entender que, si no prestamos atención, nos da la sensación de que las soluciones siempre salen desde Europa; éste sería uno de los aportes importantes de las guerra de liberación en África. En definitiva, se trataba de una guerra en la que estaban muriendo portugueses por un territorio que no les pertenecía, y esto ellos no lo podían entender. Cuando los soldados portugueses llegaban a las colonias veían una población que en la mayoría de las ocasiones no se identificaban con el proyecto colonial, ya que realmente era un proyecto colonial, aunque al final lo trataran de disimular. Pensemos que, en el mismo año 1974, en Mozambique no había prácticamente universidades; la mayoría de las universidades eran para estudiantes blancos; no había población negra. Eso llevó a los mismos portugueses a preguntarse por qué la población africana no se identificaba con ellos. Yo recuerdo haber entrevistado a un soldado portugués que había luchado en las guerras coloniales y decía: "Portugal era el espacio que ocupaba yo dentro de un coche, fuera de ese espacio Portugal se acababa". Digo esto para mostrar la dimensión de la guerra colonial y sobre todo para pensar la llamada democratización portuguesa. Se trata de una democratización que tenía como objetivo que los portugueses se pudieran definir como europeos, pero no permitía a aquellas sociedades, que compartían la condición de portugueses, tener presencia y representaciones en la historia y en la política. Este es un problema que también existe en España y en Francia, la existencia de grupos que se identifican con Francia, con Portugal, con Inglaterra o con España, pero siguen siendo identidades marcadas por el origen, o sea, son portugueses de origen caboverdiano, portugueses de origen africano, franceses de origen argelino, pero ¿por qué decimos de origen? Pues porque hay un proyecto de origen de la mayoría de los países europeos, el proyecto de Estado-nación, y es un proyecto que no está funcionando en absoluto. Se trata de un proyecto desarrollado por un grupo de poder que tiene sus referencias sobre lo que les define, que tiene sus propias referencias culturales que los une y que dan significado a ese proyecto y el resto de grupos han de asimilarse a ese proyecto; si no se asimilan quedan fuera. Todos aquellos que se quedan fuera son esas identidades de origen.

Lo que sucede en Portugal, como en otros países europeos, es que la democracia está pensada para los ciudadanos de la metrópoli y son ellos los que van a definir qué significa Portugal tras la dictadura, pero no hemos pensado cómo trasciende el legado del proyecto imperial tras el fin del colonialismo administrativo. Portugal no abrió una discusión con aquellos territorios que abandonaba, territorios de los que era expulsado. Los europeos perdieron sus colonias, pero no se han descolonizado, en el sentido de conocer y 
re-conocer, desde sus ignorancias, como plantea Boaventura de Sousa Santos, identificando esas ignorancias y ausencias y permitiendo nuevas emergencias.

PREGUNTA: Fuiste a estudiar a la Unión Soviética, en aquellos años viajaste y trabajaste en varios países de Europa. ¿Cómo te marcó la experiencia?

RESPUESTA: En primer lugar, yo creía que este sistema de colonización solo se había dado fuera de Europa, pero al llegar a la Unión Soviética descubrí que no. Cuando fui a la URSS comprendí que eso había pasado con los rusos respecto a polacos y finlandeses, con los ingleses respecto a País de Gales e Irlanda. Respecto a este tema, ahora que hablamos de colonialismo, no debemos olvidar algo muy importante, que es la cuestión de la lengua. En varios textos constitucionales de la Corona de Inglaterra se establece que el inglés se declara la lengua oficial del Imperio al mismo tiempo en la India y en País de Gales. La cuestión aquí es que puedes hablar cuatro horas al día ingles en la escuela, pero el resto de tu vida la haces en otro idioma, por lo tanto ¿cómo vas a identificarte con un proyecto que no se expresa en tu lengua? Muchas veces la lengua es un instrumento de resistencia, pero no solo, también de afirmación, porque con la lengua viene la cultura y la posibilidad de desplegar otros proyectos. Esta es una de las grandes confusiones cuando hablamos hoy en día sobre colonialismo, y es reducirlo únicamente a la línea racial, pero en estos casos hablamos de Europa, donde todos son blancos, en principio. Para estas personas la separación abismal, una de las aportaciones de las epistemologías del Sur del profesor Boaventura de Sousa Santos, la constituye la serie de líneas invisibles que te cons- truyen fuera del proyecto, y en caso de estar dentro, estás como un ser cuyos conocimientos no son generalmente válidos. Tus conocimientos son válidos únicamente para País de Gales o para Andalucía, pero no son conocimientos que aporten a la totalidad, no lo son para España o para el Reino Unidos. Este es el problema y esto nos lleva a pensar que el proyecto de los Estados-nación no está funcionando. Quizás el caso en el que lo intentaron con más fuerza fue en Francia y Alemania, y tienen los resultados que hoy vemos con las formas de populismo contra los que están dentro pero que no han sido asimilados por el proyecto. Cuando miras desde fuera el proyecto colonial, te das cuenta que nosotros ya habíamos experimentado eso, el fracaso de los Estados-nación, y podríamos todos aprender de esos errores. El proyecto del Estado-nación es un proyecto imposible, crear un territorio con millones de personas, con el mismo sistema educativo, de salud, de justicia no va a funcionar porque hay otras lenguas, otras culturas, otras historias y que también existen.

PREgUNTA: Desde hace más de una década trabajas en el desarrollo de la propuesta política e intelectual de las epistemologías del Sur, junto a Boaventura de Sousa Santos y el resto del equipo del Centro de Estudios Sociales. ¿Cuál es la crítica de las epistemologías del Sur al colonialismo?

RESPUESTA: Las epistemologías del Sur tratan de dar voz, visibilidad y dignidad epistemológica a todos aquellos que fueron silenciados y negados por el colonialismo. Bueno, en nuestro caso, en el contexto africano, el colonialismo no llega a un territorio vacío de conocimientos. El colonialismo se impone, pero la gente dialoga con el colonialismo como respuesta a 
esa relación de poder, y dialoga desde sus propias experiencias. Esto te da toda una gama de diferencias, que es lo que hoy las epistemologías del Sur ponen sobre la mesa, la diversidad de proyectos epistemológicos que son la respuesta de los colonizados a los intentos de dominación por parte de los imperios. Ya sean los subaltern studies en la India, el pensamiento decolonial y el pensamiento indígena en América Latina o las discusiones del ámbito poscolonial en África. El colonialismo trato de dibujar una línea abismal como si al otro lado no hubiera nada, pero el otro lado existe, el otro escucha al colonialismo, recibe el mensaje del colonialismo y va establecer un dialogo con él. Y esta es la parte más difícil, pues ese proyecto de modernidad eurocéntrica ha sido apropiado por el otro lado, esto da una diversidad y una dispersión inmensa. Esta es una de los grandes aprendizajes del final del colonialismo administrativo en Mozambique, que nos muestra lo mucho que quedó en Mozambique del proyecto colonial tras la independencia. Hay una frase muy interesante de Eduardo Mondlane, primer presidente del Frente de Liberación de Mozambique cuando preguntaba, poco antes de ser asesinado, qué hacer en un contexto de lucha comunista en África respecto al problema de las independencias y la desaparición del Estado portugués. Francia e Inglaterra habían organizado todo para salir, pero que el colonialismo continuara, tanto desde el punto de vista epistémico como cultural. Pero las independencias conquistadas por la guerra de autodeterminación al Estado portugués llevaron al país a una situación límite. En aquellas guerras de liberación que destruyeron el país, los miembros de los partidos independentistas mantuvieron únicamente un legado, el Estado, una herencia que resultó ser muy pesada. En este sen- tido, hay una independencia política, pero hay otro proceso de descolonización más largo, que sigue aconteciendo, ¿cómo vamos a pensar con nuestras propias ideas? Lo que en el norte se define como luchas anticoloniales, para nosotros han sido las luchas nacionalistas. Las luchas anticoloniales trataron de destruir el Estado y fue algo que no funcionó. Por tanto, debemos pensar nuestra realidad desde el encuentro colonial, nuestras herencias y las herencias del encuentro, y sobre todo, qué vamos a hacer con todo esto a partir de ahora. Las luchas de liberación del tercer mundo supusieron la emergencia ontológica de los sujetos, pero no la emergencia epistémica y eso es lo que hoy persiguen las epistemologías del Sur.

PREGUNTA: En la última década has venido bastante a Andalucía y has conocido nuestra realidad, has colaborado con investigadoras y has sido invitada a Andalucía a impartir seminarios y conferencias ¿Cómo será, desde tu punto de vista, la incorporación de Andalucía a las epistemologías del sur y a los debates del pensamiento poscolonial? ¿Qué significa para ti descolonizar Europa?

RESPUESTA: Haber sido negados es parte de nuestra herencia y de lo que somos. El capitalismo intentó, desde un punto de vista epistémico y ontológico, negar el Sur. América Latina es un mal ejemplo, en el buen sentido, pues nos muestra que el colonialismo de asentamiento es quizás la forma más perversa de colonialismo, es la destrucción total, es la construcción de espacios vacíos donde se asienta población que Europa considera como humanos. Creo que esto sucedió aquí en Andalucía, por eso es tan importante estudiar lo que sucedió en Andalucía, ya que es parte del mismo proyecto: vaciar los te- 
rritorios, rellenar con nueva población y nuevos saberes y formar a la población que permanece a que desaparezca epistémicamente y a asimilarse a esos nuevos saberes. Pero también en Andalucía pese a todas las presiones hay grupos que siguen con su memoria, que es una historia alternativa, memorias que desafían a la historia como proyecto político y como proyecto colonial.

Para mi descolonizar significar sobre todo re-conocer, reconocer la humanidad en el otro, recuperar la acogida del otro, reconociendo su humanidad, es decir, humanizar al otro de nuevo, que ha sido algo muy característico del sur y es algo que se ha perdido. Esta pérdida de humanidad nos lleva a la falta de reconocimiento y de solidaridad con el otro.

Otro de los problemas centrales en la descolonización de Europa tiene que ver con la noción de escala en el análisis político. La escala privilegiada por Europa en el análisis político ha sido el Estado-nación. En la primera mitad del siglo XX, y sobre todo en los años cuarenta, el mundo se estudiaba desde una gran diversidad de modelos políticos siendo el Estado-nación tan uno de los modelos. Tras la segunda guerra mundial, tan solo quedó el Estado como escala en los análisis políticos, pero después de los años ochenta, el análisis del Estado se redujo a su forma de Estado-nación, como si Estado fuera sinónimo de nación. Esto creo que ha sido un ejercicio de construcción de ausencias y es parte del debate en los estados africanos que no saben cómo gestionar asuntos de Estado, como las fronteras o la plurinacionalidad, desarrollando en muchos casos proyectos de Estado que no tienen demasiadas conexiones con la población local. El Estado ha sido, en el desarrollo de los proyectos coloniales desde la Conferen- cia de Berlín, la toma efectiva del poder y de la administración. Es el Estado el que transforma a las autoridades existentes en autoridades tradicionales o locales sobre las que se superpone el poder del Estado. ¿Cómo se articulan las diferentes autoridades, legitimidades locales, identidades y culturas bajo un solo Estado? Otra cuestión que me preocupa es la consideración de que el Estado es la única autoridad política legítima. Una vez más, se privilegia el Estado desde una mirada evolucionista. En diferentes ámbitos, como la antropología, se ha estudiado otras formas de autoridad política, y creo que en algún sentido sería interesante poner en diálogo varias sociedades africanas con los anarquistas españoles, me refiero al anarquismo español del primer tercio del siglo XX. Un diálogo intercultural no jerárquico de sociedades que rechazan el Estado sin jerarquizar ni desperdiciar experiencias, ya que son formas que nos ayudan a pensar nuevas formas de autoridad en las que la soberanía no recaiga sobre la violencia del Estado y, sobre todo, pensar formas de autoridad política que no sigan produciendo extranjeros en su propio territorio. Por ejemplo el caso de los vascos en el Estado español. Un vasco no puede hablar euskera en Madrid, pero una persona de Madrid si tiene derecho a ser entendido en castellano en el País Vasco.

Para mí, el problema cuando hablamos de Europa es que nos referimos a ella como centro. Siempre existe un centro, pero el centro lo creamos nosotros con nuestras propias referencias y nuestros referentes analíticos siempre proceden de un determinado lugar, en este caso, de ese proyecto eurocéntrico que está en todo el mundo. Por lo tanto, seguimos jerarquizando y privilegiando en nuestra forma de pensar esos referentes que vienen de 
Europa. En ese sentido, quizás la universidad sea el peor instrumento para la descolonización, ya que todas las universidades siguen privilegiando una cierta forma de pensar y jerarquizar entre el Derecho y el derecho indígena, por ejemplo, entre la Cultura y las culturas, entra la Lengua y las hablas. Las luchas de descolonización eran también luchas por la auto-determinación y por la capacidad de auto-definirse. Yo creo que hubo una afirmación por parte del colonialismo en el desarrollo de las universidades eurocéntricas en el sur, por ejemplo, en el contexto africano, donde desplazaron las lenguas locales, los derechos de las comunidades. Hay casos como Namibia donde se permite estudiar en la legua local, pero hay casos como Mozambique que recoge la plurinacionalidad en su constitución, pero en cambio, sigue jerarquizando el derecho público de raíz eurocéntrica y desplazando los derechos tradicionales. Nosotros no estamos quizás entrenados para pensar sobre esto, porque en nuestra facultades y universidades no nos prepararon para pensar de otra forma. Por ejemplo, toda la tradición de teoría académica nos manda a nuestros países a hacer trabajo de campo con las implicaciones coloniales que tiene el propio concepto. Y no es un problema solo de Portugal o de España, sino de la universidad como centro de saber del Estado moderno. Por eso, desde las epistemologías del Sur hemos puesto el énfasis en la importancia de descolonizar la Universidad para crear nuevos marcos de pensamiento junto a los estudiantes y jóvenes investigadores que no están aún tan contaminados del pensamiento eurocéntrico hegemónico.

PREGUNTA: Tú misma tienes formación de historiadora. Ahora mismo esta entrevista la estamos desarrollando en la Facultad de Filosofía y Letras de la Universidad de Granada, uno de los grandes centros de investigación académica y enseñanza universitaria de Historia en Andalucía, pero quizás también del sur de Europa. Al mismo tiempo, se trata de una enseñanza y una mirada de la historia profundamente eurocéntrica que privilegia los estudios en la llamada Reconquista, así como una cierta Historia Universal que tiene como centro la experiencia Europa para estudiar la historia antigua, medieval, moderna y contemporánea ¿Cómo podríamos pensar históricamente desde el sur?

RESPUESTA: Bueno, en primer lugar, tenemos que abordar el problema de los continentes, ya que es un problema de raíz eurocéntrica. La invención de Europa, la invención de África y la invención de América. Todo conocimiento geográfico tiene un centro, y ese centro siempre es un centro epistemológico. Yo, por ejemplo, en Mozambique no puedo hablar de Arabia Saudí como Próximo Oriente, no es próximo ni está al oriente, o por ejemplo me parece igualmente problemático el concepto de África subsahariana. Por lo tanto, en primer lugar hay un problema con la geografía, y es que se trata de una disciplina que genera desconocimiento. En segundo lugar, existe una forma de clasificación construida por la Ilustración como proyecto no-religioso de comprensión del mundo donde se pudiera incluir todo el mundo. Para mí, el Islam o el Cristianismo no son solo religiones, sino formas de ser. El hecho de que haya países cristianos que hayan transitado al ateísmo no significa que hayan abandonado su forma religiosa de ser y estar en el mundo. La religiosidad está tan dentro de nosotros y nosotras que es muy problemático actuar como si no existiera. Yo estudié en la Unión Soviética y casi cada año tenía un examen el día 25 de diciembre. En Mozambique, el Estado es mucho más 
ateo que los Estados europeos, por lo que todas las religiones tienen sus festividades, además las festividades del mundo islámico se basan en un calendario lunar. Una compañera me contaba que en una clase de Historia con estudiantes chinos, uno de ellos levantó la mano y preguntó, - perdone, ¿quién es Cristo? ¿A qué se refiere cuando dice "antes de que naciera Cristo"? El problema es que cada imperio asume, normaliza y uniformiza sus propias características culturales, filosóficas, geográficas y secuencias evolutivas. Estas características son naturalizadas y normalizadas en el seno de cada imperio, y son estas mismas características las que hay que desnaturalizar para volver a las viejas y a las nuevas historias. Desnaturalizar estas secuencias evolutivas y sus teleologías históricas es imprescindible para pensar de nueva una Historia no eurocéntrica. Yo recuerdo cuando era estudiante en Mozambique y llegó el comunismo en el año 1974, llegó también a las universidades y las facultades de Historia el debate sobre el feudalismo y se discutía sobre las etapas de la Historia y el "feudalismo africano" y en ese momento nosotros no nos encontrábamos. Encontrarnos implica también encontrar categorías que expliquen nuestra propia experiencia histórica, una experiencia histórica que nos entienda a nosotros mismo como centro.

Otro elemento problemático de la forma eurocéntrica de estudiar la Historia, y esto es algo que aquí en Andalucía es importante para pensar vuestra propia Historia, es que se ha privilegiado el desarrollo de las conquistas y la guerra frente a los contactos culturales, comerciales y mercantiles. Las conexiones comerciales son mucho más importantes de lo que nosotros pensamos. Hemos sido educados para pensar la Historia en términos de Historia de las conquistas. Las grandes narrativas han sido diseñadas en función de las guerras, y las conquistas coloniales, pero no es siempre la guerra la que condiciona el pasado. Hay jerarquizaciones y existen relaciones de poder desiguales, pero no es siempre la guerra física y la violencia militar la que condiciona los procesos históricos. Pero es más fácil estudiar los periodos históricos desde las fechas bélicas y los resultados de las conquistas y las guerras. Sinceramente, creo que no debe ser lo único que centre nuestra atención, más aún en esta región que es el mediterráneo es importante estudiar los intercambios, los flujos comerciales y de saberes, que se comunican e intercambian entre sí. Si hay una lección que el mediterráneo puede dar a Europa, es que hasta los años cuarenta el mediterráneo era un mundo en diálogo que hoy se intenta cerrar. A esto se refería también Sukarno, en el año 1955, en la Conferencia de Bandung, cuando decía que hay una línea en el mundo que va desde Gibraltar hasta el mar de Japón que divide a los administrados de los administradores.

A esto es a lo que el historiador Chakrabarty se refiere cuando habla de hacer otra Historia, con otros códigos analíticos, los que usamos para construir nuestra raíces y con los que miramos al futuro. Los códigos de la Historia hegemónica son códigos analíticos que nos limitan nuestras posibilidades de mirar al futuro. Nos dejan atrapados en proyectos políticos que son parte del pasado.

En esta nueva Historia, desde la perspectiva de las epistemologías del Sur, la ignorancia es muy importante. La ignorancia nos lleva a querer conocer el pasado, de indagar y la voluntad de querer conocer. En este sentido, yo no creo que la mayoría de los partidos políticos de Europa estén 
interesados en conocer esta Historia. Yo, por ejemplo, puedo hablar de la política de mi país. La mayoría de los partidos proceden de un grupo minoritario que trata de llevar su proyecto al resto de la sociedad. A esto se refería también Aime Cesarie cuando abandonó el Partido Comunista Francés y reprochaba a los dirigentes comunistas franceses que declarasen que la lucha anticapitalista era más importante que la lucha por la descolonización. Yo no estoy tan segura que exista una lucha política por llegar a las instituciones y que sea más importante que la lucha contra la violencia hacia las mujeres o las luchas por el reparto de la tierra. Tanto en África como en Andalucía las izquierdas han dejado de hablar de la tierra, que es una lucha muy importante en nuestros contextos, porque la tierra no es únicamente una discusión en términos de propiedad sino una tradición histórica de la que venimos, por las que muchas generaciones anteriores se han revelado y han luchado. Debemos comprender todo el desarrollo económico e histórico, no solo como experiencias del pasado sino como posibilidad de un presente diverso lleno de nuevas posibilidades. Esta es una de mis críticas a la izquierda, que, al llegar al poder, trata de "ayudarnos". ¿Pero quién ha definido lo que necesitamos? En muchas ocasiones estamos condicionados en todos los países por los plazos de la política cada vez que llegamos al poder, necesitas reproducir las condiciones para mantenerte en el poder y esto lleva, en la mayoría de las ocasiones, a reproducir proyectos políticos ya pensados y definidos.

PREGUNTA: Muchísimas gracias, Maria Paula, muchísimas gracias de todo corazón...

RESPUESTA: A vosotros... 